\title{
Vitamin D supplementation in the management of knee osteoarthritis: study protocol for a randomized controlled trial
}

Yuelong Cao ${ }^{1,3}$, Graeme Jones ${ }^{*}$, Flavia Cicuttini ${ }^{2}$, Tania Winzenberg ${ }^{1}$, Anita Wluka², James Sharman', Kay Nguo ${ }^{1}$ and Changhai Ding ${ }^{1,2^{*}}$

\begin{abstract}
Background: Osteoarthritis $(\mathrm{OA})$ is a common health issue worldwide in the aging population who are also commonly deficient in vitamin D. Our previous study suggested that higher serum 25-(OH)D levels were associated with reduced knee cartilage loss, implying that vitamin $D$ supplementation may prevent the progression of knee OA. The aim of the VItamin D Effects on OA (VIDEO) study is to compare, over a 2-year period, the effects of vitamin D supplementation versus placebo on knee structural changes, knee pain, and lower limb muscle strength in patients with symptomatic knee OA.

Methods/design: Randomised, placebo-controlled, and double-blind clinical trial aiming to recruit 400 subjects (200 from Tasmania and 200 from Victoria) with both symptomatic knee OA and vitamin D deficiency (serum [25-(OH)D] level of $>12.5 \mathrm{nmol} /$ liter and $<60 \mathrm{nmol} /$ liter). Participants will be randomly allocated to vitamin $\mathrm{D}$ supplementation (50,000 IU compounded vitamin $\mathrm{D}_{3}$ capsule monthly) or identical inert placebo group for 2 years. The primary endpoint is loss of knee cartilage volume measured by magnetic resonance imaging (MRI) and Western Ontario and McMaster Universities Index of OA (WOMAC) knee pain score. The secondary endpoints will be other knee structural changes, and lower limb muscle strength. Several other outcome measures including core muscle images and central blood pressure will be recorded. Linear and logistic regression will be used to compare changes between groups using univariable and multivariable modeling analyses. Both intention to treat and per protocol analyses will be utilized.
\end{abstract}

Discussion: The trial is designed to test if vitamin D supplementation will reduce loss of knee cartilage volume, prevent the progression of other knee structural abnormalities, reduce knee pain and strengthen lower limb muscle strength, thus modify disease progression in knee OA.

Trial registration: ClinicalTrials.gov identifier: NCT01176344; Australian New Zealand Clinical Trials Registry: ACTRN12610000495022

Keywords: Vitamin D, Osteoarthritis, Magnetic resonance imaging

\footnotetext{
* Correspondence: g.jones@utas.edu.au; Changhai.Ding@utas.edu.au

${ }^{1}$ Menzies Research Institute Tasmania, University of Tasmania, Private Bag 23,

Hobart, TAS 7000, Australia

${ }^{2}$ Department of Epidemiology and Preventive Medicine, Monash University,

Melbourne, 3004, Australia

Full list of author information is available at the end of the article
} 


\section{Background}

Osteoarthritis (OA) is the most common form of arthritis in the world and one of the most common chronic conditions managed in Australian general practice $[1,2]$. It is characterized by the gradual loss of articular cartilage and changes to other joint structures eventually leading to total joint replacement. Currently there is no cure for OA, and the development of innovative and cost-effective approaches to prevent the development and progression of $\mathrm{OA}$ is urgent and important.

Vitamin D comprises a group of fat-soluble secosteroids emcompassing two major molecules, vitamin $\mathrm{D}_{2}$ and vitamin $D_{3}$. Vitamin $D$ is circulated to the liver where it is converted to the prohormone calcidiol, or 25-hydroxy-vitamin D (25-(OH)D), which is the best indicator of vitamin D status [3-5]. Vitamin D deficiency is very common in older people. It is estimated that 20 to $100 \%$ of elderly men and women in North America and Europe are vitamin $\mathrm{D}$ deficient (mostly defined as a serum level of $25-(\mathrm{OH}) \mathrm{D}<50 \mathrm{nmol} / \mathrm{liter})[6,7]$. High rates of vitamin $\mathrm{D}$ deficiency have also been reported in all sectors of the community of Australia, especially in Tasmania and Victoria where this study will be conducted [8-10].

It has been widely recognized that $\mathrm{OA}$ is a disease affecting the whole joint, including cartilage, bone and muscle. Through targeting these joint tissues vitamin $\mathrm{D}$ supplementation may modify disease progression in OA. Vitamin D receptors (VDRs) are found in human articular chondrocytes [11], and $1 \alpha-25(\mathrm{OH})_{2} \mathrm{D}_{3}$ regulates the expression of metalloproteinase (MMP) and prostaglan$\operatorname{din} \mathrm{E}_{2}\left(\mathrm{PGE}_{2}\right)$ in chondrocytes via VDRs [11]. Vitamin D could enhance the ability of bone to respond optimally to pathophysiological processes in $\mathrm{OA}$, thus prevent disease progression $[12,13]$. Furthermore, $1,25(\mathrm{OH})_{2} \mathrm{D}$ leads to de novo protein synthesis, muscle cell growth, and improved muscle function, and thus has a beneficial effect on muscle strength [14].

Epidemiological studies have provided preliminary evidence supporting the potential use of vitamin $D$ for the treatment of OA. Lower serum levels of 25- $(\mathrm{OH}) \mathrm{D}$ were associated with greater knee pain [15] and higher prevalence of radiographic OA [16], and predicted incidence of knee pain [17], progression/incidence of radiographic OA [18,19], and loss of joint space, as well as osteophyte growth [18]. Magnetic resonance imaging (MRI) has been utilized to directly assess knee structural alterations such as cartilage volume, cartilage defects, subchondral bone changes and meniscal lesions. Using MRI, we reported that, in cross-sectional analysis, serum 25-(OH)D level was significantly and positively associated with knee cartilage volume in older men and women, and vitamin D insufficiency was positively associated with medial and lateral tibial bone area in women. Longitudinally, higher baseline serum levels of $25-(\mathrm{OH}) \mathrm{D}$ predicted reduced loss of cartilage volume over 2 years, and increases in vitamin $\mathrm{D}$ levels were associated with further protective association $[15,16]$. Furthermore, serum levels of 25- $(\mathrm{OH}) \mathrm{D}$ were also associated with increased leg muscle strength and quality, and thus may be important for the maintenance of muscle function [20].

Based on this experimental and epidemiological evidence, we have initiated a randomized, placebocontrolled trial (Vitamin D Effect on Osteoarthritis, VIDEO study) to determine if vitamin D supplementation can reduce loss of knee cartilage volume, prevent the progression of other knee structural abnormalities and strengthen lower limb muscle strength, and thus modify disease progression in knee OA. The effects of vitamin D supplementation on the progression of knee pain will also be determined.

In a sub-study, we will examine the effects of vitamin D supplementation on the function of deep lumbo-pelvic stabilising muscles. The protective deep lumbo-pelvic stabilising muscles, which include (but not exclusively) the transversus abdominus ( $\operatorname{Tr} A b)$ and lumbar multifidus and which are known as core muscles, become dysfunctional shortly after the onset of low back pain, and that ongoing muscle dysfunction is associated with persistent low back pain $[18,21]$. Muscle weakness can be a sign of vitamin D deficiency [19] and therefore, vitamin D supplementation may have beneficial effects on functionally important core muscles.

Furthermore, we will determine the effect of vitamin D supplementation on blood pressure (clinical, ambulatory, upper arm and central measures) and arterial stiffness (aortic pulse wave velocity). Several lines of evidence suggest that vitamin D deficiency may influence blood pressure via mechanisms including activation of the renin-angiotensin-aldosterone system (RAAS) [22-24]. Central and ambulatory blood pressure will be the main outcomes because central blood pressure is the actual pressure load experienced by the heart (and other organs such as the kidneys and brain) rather than the pressure at the upper arm [25], and ambulatory blood pressure is regarded as the gold standard technique because it correlates with target organ damage and provides more accurate information on daily (including night time) blood pressure fluctuations [26].

\section{Methods/design}

\section{Study design}

VIDEO is a randomized, placebo-controlled double-blind clinical trial. Four hundred subjects (200 from Tasmania and 200 from Victoria) with symptomatic knee OA and serum $25-(\mathrm{OH}) \mathrm{D}>12.5 \mathrm{nmol} /$ liter and $<60 \mathrm{nmol} /$ liter will be recruited and randomly allocated to either the 
treatment or placebo control group. Recruitment methods will include advertisements through the local media and community groups as well as liaisons with general practitioners, specialist rheumatologists, and orthopedic surgeons. Ethics approval has been received from The Tasmania Health and Human Medical Research Ethics Committee (reference number H1040) and Monash University Human Research Ethics Committee (reference number CF10/1182 - 2010000616). Informed written consent will be obtained from all participants.

\section{Inclusion criteria}

The inclusion criteria were as follows: age 50 to 79 years; symptomatic knee OA for at least 6 months with a pain at least $20 \mathrm{~mm}$ on a $100 \mathrm{~mm}$ visual analogue scale (VAS); American College of Rheumatology (ACR) criteria for symptomatic knee OA assessed by a rheumatologist [27]; ACR functional class rating of I, II and III [28]; relatively good health, with a score of 0 to 2 on a 5point Likert scale (with a range of 0 indicating very good health to 4 indicating very poor health), according to the investigators global assessment of disease status; serum $25-(\mathrm{OHD}) \mathrm{D}>12.5 \mathrm{nmol} /$ liter and $<60 \mathrm{nmol} /$ liter; able to read, speak and understand English, capable of understanding the study requirements and willing to cooperate with the study instructions.

\section{Exclusion criteria}

Exclusion criteria were as follows: severe radiographic knee OA, grade 3 according to Altman's atlas [29]; severe knee pain on standing (more than $80 \mathrm{~mm}$ on $100-\mathrm{mm}$ VAS); any contraindication to having MRI; rheumatoid or psoriatic arthritis, lupus or cancer; severe cardiac or renal impairment; hypersensitivity to vitamin D; any condition possibly affecting oral drug absorption (for example, gastrectomy or malabsorption syndromes); significant trauma to knees, including arthroscopy or significant injury to ligaments or menisci of the knee within one preceding the study; anticipated need for knee or hip surgery within the next 2 years; history of taking vitamin D supplements within the previous 30 days; history of taking an investigational drug within the previous 30 days.

\section{Randomization}

Participants in each site will be randomly assigned to the intervention arm or placebo arm in a ratio of 1:1 and the randomization will be double-blind. Allocation of participants will be based on computer-generated random numbers. Allocation concealment will be ensured by the use of an identical inert placebo, and a central automated allocation procedure, with security in place to ensure allocation data cannot be accessed or influenced by any person.

\section{Intervention}

Participants in the intervention arm will take one capsule per month of 50,000 IU (1.25 mg) of a vitamin D3 compound (cholecalciferol), purchased from Nationwide Compounding Pharmacy, Melbourne, Australia[30], and patients in the control arm will receive an identical inert placebo provided by the same company. Patients are required to record their medication information in personal diaries and a reminder will be given each month. All participants will receive the recommended standard of care. The duration of the study is 2 years.

\section{Quality assurance}

To ensure that this trial will be of a high standard and delivered in accordance with the trial protocol, all research staff will be provided with a standard protocol and case report form, and will be trained to competently administer items as per protocol. The investigators, research assistants and outcome assessors are different people. Protocols will not be altered during the study timeframe.

\section{Outcome measures}

The co-primary efficacy endpoints of the study will be MRI assessment of volume changes in knee cartilage from baseline to month 24, as well as the Western Ontario and McMaster Universities Index of $\mathrm{OA}$ (WOMAC) score [31] (Table 1). The secondary endpoints will be other knee structural changes (cartilage defects, tibial plateau bone area, and bone marrow lesions, meniscal tear and extrusion) from baseline to month 24, and lower limb muscle strength at months 3 , 6, 12 and 24 (Table 1).

\section{MRI assessment of knee structural changes}

Knees will be imaged in the sagittal plane on a 1.5-T whole body MRI unit using a commercial transmitreceive extremity coil. Fat-saturated T1-weighted spoiled gradient echo (GRE) and T2-weighted/proton densityweighted fast spin echo (FSE) sequences will be used. The images will be assessed by two readers blinded to the treatment according to the methods described in our previous publications [15,32].

Cartilage volume: the volumes of individual cartilage plates (medial tibial, lateral tibial and patella) are isolated from the total volume by manually drawing disarticulation contours around the cartilage boundaries on a section-by-section basis. Sagittal images will be obtained at a partition thickness of $1.5 \mathrm{~mm}$ and an in-plane resolution of $0.31 \times 0.31 \mathrm{~mm}(512 \times 512$ pixels $)$, then resampled by means of bilinear and cubic interpolation (area of $312 \mu \mathrm{m} \times 312 \mu \mathrm{m}$ and multiplied by $1.5 \mathrm{~mm}$ thickness, continuous sections) for the final 3D rendering. Particular cartilage volume was then determined by 
Table 1 Timetable and measures to be made

\begin{tabular}{|c|c|c|c|c|c|c|}
\hline & \multirow[t]{2}{*}{ Screening } & \multicolumn{5}{|c|}{ Month(s) } \\
\hline & & 0 & 3 & 6 & 12 & 24 \\
\hline \multicolumn{7}{|l|}{ Co-primary outcome measure } \\
\hline MRI (cartilage volume changes) & & $\checkmark$ & & & & $\checkmark$ \\
\hline WOMAC & & $\checkmark$ & $\checkmark$ & $\checkmark$ & $\checkmark$ & $\checkmark$ \\
\hline \multicolumn{7}{|l|}{ Secondary outcome measure } \\
\hline MRI (other structural changes) & & $\checkmark$ & & & & $\checkmark$ \\
\hline Lower limb muscle strength & & $\checkmark$ & $\checkmark$ & $\checkmark$ & $\checkmark$ & $\checkmark$ \\
\hline \multicolumn{7}{|l|}{ Other measures } \\
\hline Core musculature measure & & $\checkmark$ & & & $\checkmark$ & $\checkmark$ \\
\hline Hand grip strength & & $\checkmark$ & $\checkmark$ & $\checkmark$ & $\checkmark$ & $\checkmark$ \\
\hline Central and upper arm blood pressure & & $\checkmark$ & & $\checkmark$ & $\checkmark$ & $\checkmark$ \\
\hline Aortic stiffness & & $\checkmark$ & & $\checkmark$ & $\checkmark$ & $\checkmark$ \\
\hline Physical activity (IPAQ) & & $\checkmark$ & & & & $\checkmark$ \\
\hline Body fat & & $\checkmark$ & & & $\checkmark$ & $\checkmark$ \\
\hline Low foot pain & & $\checkmark$ & $\checkmark$ & $\checkmark$ & $\checkmark$ & $\checkmark$ \\
\hline Low back pain & & $\checkmark$ & & $\checkmark$ & $\checkmark$ & $\checkmark$ \\
\hline Depression & & $\checkmark$ & $\checkmark$ & $\checkmark$ & $\checkmark$ & $\checkmark$ \\
\hline Quality of life & & $\checkmark$ & & $\checkmark$ & $\checkmark$ & $\checkmark$ \\
\hline Previous knee injury and occupation & & $\checkmark$ & & & & $\checkmark$ \\
\hline Weight & & $\checkmark$ & $\checkmark$ & $\checkmark$ & $\checkmark$ & $\checkmark$ \\
\hline Height & & $\checkmark$ & & & & $\checkmark$ \\
\hline Girth measurements & & $\checkmark$ & & & $\checkmark$ & $\checkmark$ \\
\hline Knee radiograph & $\checkmark$ & & & & & \\
\hline Serum 25-(OH)D & $\checkmark$ & & $\checkmark$ & & & $\checkmark$ \\
\hline Serum calcium, phosphate, creatinine & $\checkmark$ & & $\checkmark$ & & & \\
\hline Sun exposure & & $\checkmark$ & & $\checkmark$ & $\checkmark$ & $\checkmark$ \\
\hline Cigarette smoking & & $\checkmark$ & & & & $\checkmark$ \\
\hline Diet (FFQ) and pedometer & & $\checkmark$ & & & & $\checkmark$ \\
\hline Medications & $\checkmark$ & $\checkmark$ & $\checkmark$ & $\checkmark$ & $\checkmark$ & $\checkmark$ \\
\hline Pill counts and adverse events & & $\checkmark$ & $\checkmark$ & $\checkmark$ & $\checkmark$ & $\checkmark$ \\
\hline
\end{tabular}

Participants who withdraw within one year will be asked to have MRI at month 12; patients who withdraw after one year will be asked to have MR straight away. MRI: magnetic resonance imaging; WOMAC: Western Ontario McMaster Universities Osteoarthritis Index; 25-(OHD)D: 25-hydroxy-vitamin D; FFQ: food frequency questionnaire.

summing all the pertinent voxels within the resultant binary volume.

Cartilage defects assessment: the cartilage defects $(0$ to 4) will be graded at medial tibial and femoral, lateral tibial and femoral, and patellar sites: grade 0 , normal cartilage; grade 1 , focal blistering and intracartilaginous lowsignal intensity area with an intact surface and bottom; grade 2, irregularities on the surface or bottom and loss of thickness of less than 50\%; grade 3, deep ulceration with loss of thickness of more than 50\%; grade 4, fullthickness chondral wear with exposure of subchondral bone.
Knee tibial plateau bone area: the area of the medial and lateral tibial plateau bone will be measured manually on the three reformatted images closest to the tibial cartilage. An average of these three areas will be used as an estimate of the tibial plateau bone area.

Subchondral bone marrow lesions: This will be assessed on the T2-weighted MRI and defined as discrete areas of increased signal adjacent to the subcortical bone at the lateral, medial femur and/or tibia. Each bone marrow lesion will be scored on the basis of lesion size, for example, a lesion is scored as grade 1 if it occupies $<25 \%$ of the region; grade 2 if it occupies $25 \%$ to $50 \%$ of the region; or grade 3 if it occupies $>50 \%$ of the region.

Meniscal tear assessment: the menisci will be assessed in the sagittal view and confirmed in the coronal and axial views as previously described [33]. In brief, the presence or absence of a tear is based on the presence of a signal, which is line shaped, brighter than the dark meniscus, and reaches the surface of the meniscus at both ends within six defined regions (anterior horn, body, and posterior horn at both medial and lateral tibiofemoral compartments).

Mensical extrusion assessment: the extent of meniscal extrusion on the medial or lateral edges of the tibial femoral joint space for the anterior, body, and posterior horns of the menisci will be graded, where a score of $0=$ no extrusion, $1=$ partial meniscal extrusion, and $2=$ complete meniscal extrusion with no contact with the joint space.

\section{Lower limb muscle strength}

This will be measured by dynamometry (TTM Muscle Meter, Tokyo, Japan) at the lower limb (involving both legs simultaneously). The muscles measured with this technique are mainly the quadriceps and hip flexors. The device will be calibrated by suspending known weights at regular intervals.

\section{WOMAC}

Knee pain will be assessed by both WOMAC pain subscale (walking on a flat surface, going up/down stairs, at night in the bed, sitting/lying and standing upright) and a $100 \mathrm{~mm}$ VAS.

\section{Other measurements}

Core musculature measure: Core muscle images will be taken at baseline and 12 months. Images of the core muscles (TrAb, internal oblique muscles and LM) are taken with real-time dynamic ultrasound using a fully featured big box diagnostic ultrasound machine (Phillips HDI 5000, Bothell, WA, US) with a hand held $7.5 \mathrm{mHz}$ linear array transducer. Images are taken of right and left sides, both at rest and during contraction (drawing in of abdomen) using previously published protocols $[34,35]$. 
These measures have a high degree of reliability with an interclass correlation coefficient (ICC) $>0.90$ across a range of studies [36].

Upper arm blood pressure, central blood pressure and aortic stiffness: Clinical upper arm blood pressure will be measured twice after 5 minutes seated rest using a validated device (Omron HEM-907, Kyoto, Japan). Seated clinical central blood pressure will be recorded (immediately after upper arm blood pressure) using radial applanation tonometry (SphygmoCor 8.1, AtCor Medical, Sydney, Australia). Aortic stiffness will be measured by electrocardiogram-gated, sequential carotid to femoral pulse wave velocity as per expert consensus [37].

Physical activity: Physical activity will primarily be assessed using a pedometer (SW 200 Digi-Walker, Yamax Corporation, Tokyo, Japan), which measures vertical displacement (steps per day). The pedometer will be worn for seven consecutive days on two occasions (baseline and 2 years) as up to seven days is required to accurately assess habitual physical activity [38]. We will also measure physical activity using the International Physical Activity Questionnaire (IPAQ) short version [39].

Body fat: Body fat will be assessed using bioelectrical impedance analysis (BIA) (BIA analyser, Quantum II, RJL Systems, Michigan, USA). Fat-free mass, \% fat-free mass, fat mass and \% fat mass will be assessed [40].

Hand grip strength: Hand grip strength will be assessed to the nearest $\mathrm{kg}$ in both the right and left hand using a hydraulic hand dynamometer (Saehan Corporation, Masan, Korea). Both hands will be alternately measured in triplicate.

Radiographic OA: this will be assessed at baseline by a standing semiflexed anterior-posterior (AP) radiograph as per the Altman atlas [29]. Radiographs will also be assessed simultaneously by two observers using the Osteoarthritis Research Society International (OARSI) atlas to score osteophytes and joint space narrowing on a four-point scale (0 to 3 ).

Laboratory measurements: serum 25-(OH)D will be assayed at month 0, 3 and 24, utilizing a Liquid Phase radioimmunoassay (Immunodiagnostics Systems Ltd, Boldon, Tyne \& Wear, UK). Serum calcium, phosphate and renal function will be assessed at month 0 and 3 using routine biochemical methods.

Anthropometrics and other questionnaires: Height will be measured to the nearest $0.1 \mathrm{~cm}$ (with shoes removed) using a stadiometer (Leicester Height Measure, Invicta Plastics Ltd, Leicester, UK). Weight will be measured to the nearest $0.1 \mathrm{~kg}$ (with shoes and bulky clothing removed) using electronic scales (Heine S-7307, Heine, New Hampshire, USA). Waist and hip measurements will be assessed using a tape measure to the nearest $0.1 \mathrm{~cm}$ (Figure Finder Tape Measure, Novel Products
Inc, Illinois, USA). Sun exposure, employment status and occupation, depression, smoking status, previous knee injury, dietary intake, low back and foot pain and quality of life will be assessed by questionnaires.

\section{Safety assessments}

Spontaneously reported adverse events will be recorded throughout the study. Intensity and relationship with the study medication will be ascribed.

\section{Sample size}

All sample size calculations assume $\alpha=0.05$ and $\beta=0.20$ and are performed based upon formulae provided by Cohen [41]. Table 2 describes the sample size (each arm) needed to detect the specified differences between the placebo and vitamin D arms with at least $80 \%$ power for each outcome.

Previous studies, including our own, suggest that OA patients have a loss of cartilage volume of 4 to $5 \%$ per year at different joint sites, respectively [42]. Vitamin D supplementation in doses ranging from 400 to $800 \mathrm{IU} / \mathrm{d}$ increased the serum level of $25-(\mathrm{OH}) \mathrm{D}$ by $27 \mathrm{nmol} / \mathrm{liter}$ per year in 7,964 men and women from five studies [43]. We estimate from our published data [15] that this change will lead to absolute reduction in loss of cartilage volume by $2.2 \%$ at the medial tibial site after vitamin D supplementation. The sample size that is needed to detect this difference is calculated (Table 2).

We have shown that male OA patients have an increase in medial tibial bone area of $1.6 \pm 2.8 \%$ per year [44], and an incidence of knee cartilage defects of $80 \%$ over 2 years [45]. There are no data known to the investigators about the associations between change in vitamin $\mathrm{D}$ and change in bone area or cartilage defects. However, healthy subjects have been shown to have an increase in tibial bone area of $0.7 \%$ per year (CD et al., unpublished), and an incidence of knee cartilage defects of $65 \%$ over 2 years in older people [46]. Assuming that changes in cartilage defects and bone area in $\mathrm{OA}$ patients will be suppressed by vitamin $\mathrm{D}$ supplementation to the levels in the healthy subjects, the sample size needed to detect these differences is given in Table 2.

Table 2 Sample size calculation

\begin{tabular}{lccc}
\hline & $\begin{array}{c}\text { Mean } \\
\text { (SD) }\end{array}$ & $\begin{array}{c}\text { Detectable } \\
\text { difference }\end{array}$ & $\begin{array}{c}\text { Calculated } \\
\text { sample size } \\
\text { (per arm) }\end{array}$ \\
\hline $\begin{array}{l}\text { Loss of volume of medial } \\
\text { tibial cartilage }\end{array}$ & $4.5 \% \pm 6.5 \%$ & $2.16 \%$ & 143 \\
$\begin{array}{l}\text { Increase in medial tibial } \\
\text { bone area }\end{array}$ & $1.6 \% \pm 2.8 \%$ & $0.9 \%$ & 153 \\
$\begin{array}{l}\text { Incidence of knee } \\
\text { cartilage defects }\end{array}$ & $80 \%$ & $15 \%$ & 136 \\
\hline
\end{tabular}


Therefore, 200 patients in each arm (allowing for a $20 \%$ dropout over the trial) will be sufficient to detect the differences between treatment groups.

\section{Analysis plan}

Statistical primary comparisons for total and subscale WOMAC scores will be made using a repeated measures mixed model with terms for treatment, month, center and the corresponding baseline values as the covariates. The independent $t$-tests will be used to compare changes between groups in quantitative data from baseline to the end of follow-up. Linear regression (annual changes in cartilage volume, cartilage defects, bone area and muscle strength as the dependent variables, and treatment as the independent variable) and logistic regression (development/progression of bone marrow lesions and meniscal abnormalities as the dependent variables, treatment as the independent variable) analyses will be applied in univariate and multivariate modeling adjusted for age, sex, body mass index, baseline $25-(\mathrm{OH}) \mathrm{D}$ and other disease status.

In secondary analysis of loss in cartilage volume, the minimal clinically important differences (MCID) in cartilage volume will be calculated [47] and logistic regression will be used to determine the association between cartilage loss (> = MCID vs. <MCID) and treatment before and after adjustment for the covariates described above.

Both intention to treat and per protocol analyses will be utilized. Per protocol will be defined as achieving a $25-(\mathrm{OH}) \mathrm{D}$ level $>60 \mathrm{nmol} / \mathrm{liter}$ at month 3 . The last observation carried forward method will be used in the analysis of all outcomes among patients who made at least one follow-up visit but did not complete the whole study.

\section{Data integrity and management}

All data obtained will be kept strictly confidential and will be stored electronically on a database with secured and restricted access. Data transfer will be encrypted and any information capable of identifying individuals will be removed.

\section{Withdrawal}

If a participant withdraws or is removed from the study, the reason and date of discontinuation will be recorded. Any participant who withdraws within year 1 will be asked to have MRI at the end of year 1; participants withdrawing after year 1 will be asked to have MRI on leaving the study.

\section{Monitoring}

The trial will be overseen and monitored by a project manager. The project manager will visit each site to examine trial procedures to ensure data quality and compliance with the trial protocol.

\section{Discussion}

We have proposed this protocol to determine if vitamin D supplementation can slow disease progression in patients with knee OA. Vitamin D may have beneficial effects for the treatment of OA, although there are currently no recommended guidelines for this approach [48]. Hence, well-designed randomized controlled trials are required to test if vitamin $\mathrm{D}$ has disease-modifying and pain-relieving effects. Such studies also need an appropriate follow-up period to capture joint structural changes using objective measurements over the course of $\mathrm{OA}$, and this has been incorporated into the design of the VIDEO study.

Assessing disease-modifying effects on OA requires an accurate measurement tool that is able to evaluate improvements in cartilage and joint health. Radiographic assessment of $\mathrm{OA}$ is two-dimensional, lacks sensitivity for changes over a short period and is highly susceptible to measurement error through factors such as variation in joint positioning [49]. MRI allows direct, accurate and reliable assessment of joint structural changes over time. These structural changes include cartilage loss, cartilage defects, increased tibial bone area, subchondral bone marrow lesions, meniscal tears and meniscal extrusion. Almost all these structural changes are predictive of total knee replacement $[45,50,51]$, suggesting they are clinically relevant. Simultaneously, we will assess change in knee pain over time using WOMAC as a co-primary endpoint. Thus, the findings from this study will show whether vitamin D supplementation has both diseasemodifying and symptom-relieving effects.

As suggested by the 2011 Endocrine Society Clinical Practice Guideline, all adults aged 50 to 70 years, and those over 70 years old require at least 600 and $800 \mathrm{IU} / \mathrm{d}$ of vitamin $\mathrm{D}$ respectively, to maximize bone health and muscle function. To raise blood levels of 25-(OH)D above $75 \mathrm{nmol} /$ liter (the lowest sufficient threshold) requires at least 1500 to $2000 \mathrm{IU} / \mathrm{d}$ of supplemental vitamin D [52]. Thus, our study design provides a dose of 50,000 IU monthly to achieve serum 25-(OH)D levels above $60 \mathrm{nmol} /$ liter in all compliant subjects [53]. This method will be less costly and will be more convenient than daily treatment. Toxicity is extremely unlikely with this dose.

Two sub-studies will simultaneously be included in this trial. Firstly, we will examine the effects of vitamin $\mathrm{D}$ on the function of the deep lumbo-pelvic stabilizing muscles. Besides implications for low back pain, in healthy people core muscles have also been implicated in varying aspects of physical function. The lateral abdominal muscles are theorized to control movement and provide stability to the trunk for functional activities and this is supported in a number of studies [54]. Vitamin D supplementation may have beneficial effects on 
functionally important core muscles. Secondly, we will determine the effect of vitamin D supplementation on blood pressure and aortic stiffness. A recent systematic review suggested that there is accumulating evidence to support the hypothesis that vitamin D deficiency contributes to hypertension, and randomized controlled trials (RCTs) are greatly needed to clarify and to definitively prove the effect of vitamin D on blood pressure [55]. The VIDEO study aims to be the first to assess this.

In summary, knee OA is a major, but poorly understood, public health problem. Vitamin D deficiency may play a role in the progression of $\mathrm{OA}$, and based on our novel preliminary data, the VIDEO study has been designed to determine whether intervening with vitamin $\mathrm{D}$ supplementation can in fact slow the progression of this disease and relieve knee pain. If correcting vitamin $\mathrm{D}$ deficiency can reduce rates of cartilage loss to lower levels as seen in older people without OA, it will significantly prolong the time it takes to reach end-stage OA eventually requiring joint replacement. This suggests great potential for substantial cost savings through reductions in joint replacement surgery, as well as potential for great improvements in the quality of life for people with OA. The success of this study will provide scientific evidence for using a cost-effective and innovative approach to addressing this clinically significant problem and will lend itself to an easy public health intervention.

\section{Trial status}

Upon submission, VIDEO study is in the process of patient recruitment.

\footnotetext{
Abbreviations

25-(OH)D: 25-hydroxy-vitamin D; ACR: American College of Rheumatology; AQOL: assessment of quality of life; BIA: bioelectrical impedance analysis; CRF: case report form; FSEL: fast spin echo; FFQ: food frequency questionnaire; GRE: gradient echo; ICC: interclass correlation coefficient; IPAQ: International Physical Activity Questionnaire; LM: lumbar multifidus; MCID: minimal clinically important differences; MMP: metalloproteinase; MRI: magnetic resonance imaging; OA: osteoarthritis; OARSI: Osteoarthritis Research Society International; PHQ-9: Personal Health Questionnaire Depression Scale-9; $\mathrm{PGE}_{2}$ : prostaglandin $\mathrm{E}_{2}$; RAAS: renin-angiotensinaldosterone system; RCT: randomized controlled trial; SOP: standard operating procedure; TASOAC: Tasmania Older Adult Cohort;

TrAb: transversus abdominus; VAS: visual analogue scales; VIDEO: Vitamin D Effects on Osteoarthritis; VDR: vitamin D receptors; WOMAC: Western Ontario McMaster Universities Osteoarthritis Index.
}

\section{Competing interests}

The authors declare that they have no competing interests.

\section{Authors' contributions}

CD and GJ conceived the study, CD, GJ, FC, TW, AW, JS, KN and YC participated in its design and coordination, and performed the research. $Y C$, $G J, K N$ and CD drafted the manuscript. All authors revised the manuscript and gave final approval of the version to be submitted.

\section{Funding}

The VIDEO study is supported by the National Health \& Medical Research Council (NHMRC ID 605501).

\section{Acknowledgements}

Jodi Barling, Judy Hankin and Alice Noone have been involved in the coordination of this study.

\section{Author details}

'Menzies Research Institute Tasmania, University of Tasmania, Private Bag 23, Hobart, TAS 7000, Australia. ${ }^{2}$ Department of Epidemiology and Preventive Medicine, Monash University, Melbourne, 3004, Australia. ${ }^{3}$ Research Institute of Orthopaedics, Shuguang Hospital Affiliated to Shanghai University of Traditional Chinese Medicine, Shanghai 201203, China.

Received: 4 April 2012 Accepted: 18 July 2012

Published: 6 August 2012

\section{References}

1. Felson DT: Clinical practice. Osteoarthritis of the knee. N Engl J Med 2006, 354:8410-8848.

2. Brand C, Hunter D, Hinman R, March L, Osborne R, Bennell K: Improving care for people with osteoarthritis of the hip and knee: how has national policy for osteoarthritis been translated into service models in Australia? Int J Rheum Dis 2011, 14:181-190.

3. Bjelakovic G, Gluud LL, Nikolova D, Whitfield K, Wetterslev J, Simonetti RG, Bjelakovic M, Gluud C: Vitamin D supplementation for prevention of mortality in adults. Cochrane Database Syst Rev 2011, :CD007470.

4. Holick MF: Vitamin D status: measurement, interpretation, and clinical application. Ann Epidemiol 2009, 19:73-78.

5. Carter GD: Accuracy of 25-hydroxyvitamin D assays: confronting the issues. Curr Drug Targets 2011, 12:19-28.

6. Holick MF: Vitamin D deficiency. N Engl J Med 2007, 357:266-281.

7. Greene-Finestone LS, Berger C, de Groh M, Hanley DA, Hidiroglou N, Sarafin K, Poliquin S, Krieger J, Richards JB, Goltzman D: 25-Hydroxyvitamin D in Canadian adults: biological, environmental, and behavioral correlates. Osteoporos Int 2011, 22:1389-1399.

8. van der Mei IA, Ponsonby AL, Dwyer T, Blizzard L, Taylor BV, Kilpatrick T, Butzkueven H, McMichael AJ: Vitamin D levels in people with multiple sclerosis and community controls in Tasmania, Australia. J Neurol 2007, 254:581-590.

9. Erbas B, Ebeling PR, Couch D, Wark JD: Suburban clustering of vitamin D deficiency in Melbourne, Australia. Asia Pac J Clin Nutr 2008, 17:63-67.

10. van der Mei IA, Ponsonby AL, Engelsen O, Pasco JA, McGrath JJ, Eyles DW, Blizzard L, Dwyer T, Lucas R, Jones G: The high prevalence of vitamin D insufficiency across Australian populations is only partly explained by season and latitude. Environ Health Perspect 2007, 115:1132-1139.

11. Tetlow LC, Woolley DE: Expression of vitamin D receptors and matrix metalloproteinases in osteoarthritic cartilage and human articular chondrocytes in vitro. Osteoarthritis Cartilage 2001, 9:423-431.

12. McAlindon TE: Nutraceuticals: do they work and when should we use them? Best Pract Res Clin Rheumatol 2006, 20:99-115.

13. Holick MF: High prevalence of vitamin D inadequacy and implications for health. Mayo Clin Proc 2006, 81:353-373.

14. Bischoff-Ferrari HA, Giovannucci E, Willett WC, Dietrich T, Dawson-Hughes B: Estimation of optimal serum concentrations of 25 -hydroxyvitamin $D$ for multiple health outcomes. Am J Clin Nutr 2006, 84:18-28.

15. Ding C, Cicuttini F, Parameswaran V, Burgess J, Quinn S, Jones G: Serum levels of vitamin $D$, sunlight exposure, and knee cartilage loss in older adults: the Tasmanian older adult cohort study. Arthritis Rheum 2009, 60:1381-1389.

16. Ding C, Cicuttini F, Jones G: Tibial subchondral bone size and knee cartilage defects: relevance to knee osteoarthritis. Osteoarthritis Cartilage 2007, 15:479-486.

17. Laslett L, Ding C, Quinn S, Burgess J, Parameswaran V, Winzenberg T, Jones G: Serum 25 Hydroxy Vitamin D (25OHD) and Incident or Worsening Knee Pain In Older Adults: A Five Year Longitudinal Study [abstract]. Arthritis Rheum 2011, 63(Suppl 10):876.

18. Hodges PW, Richardson CA: Inefficient muscular stabilization of the lumbar spine associated with low back pain. A motor control evaluation of transversus abdominis. Spine (Phila Pa 1976) 1996, 21:2640-2650.

19. Glerup H, Mikkelsen K, Poulsen L, Hass E, Overbeck S, Andersen H, Charles P, Eriksen EF: Hypovitaminosis D myopathy without biochemical signs of osteomalacic bone involvement. Calcif Tissue Int 2000, 66:419-424. 
20. Scott D, Blizzard L, Fell J, Ding C, Winzenberg T, Jones G: A prospective study of the associations between 25-hydroxy-vitamin D, sarcopenia progression and physical activity in older adults. Clin Endocrinol (Oxf) 2010, 73:581-587.

21. Hides JA, Richardson CA, Jull GA: Multifidus muscle recovery is not automatic after resolution of acute, first-episode low back pain. Spine (Phila Pa 1976) 1996, 21:2763-2769.

22. Li YC, Kong J, Wei M, Chen ZF, Liu SQ, Cao LP: 1,25-Dihydroxyvitamin D(3) is a negative endocrine regulator of the renin-angiotensin system. $J$ Clin Invest 2002, 110:229-238.

23. Simpson RU, Hershey SH, Nibbelink KA: Characterization of heart size and blood pressure in the vitamin D receptor knockout mouse. J Steroid Biochem Mol Biol 2007, 103:521-524.

24. Xiang W, Kong J, Chen S, Cao LP, Qiao G, Zheng W, Liu W, Li X, Gardner DG, Li YC: Cardiac hypertrophy in vitamin D receptor knockout mice: role of the systemic and cardiac renin-angiotensin systems. Am J Physiol Endocrinol Metab 2005, 288:E125-E132.

25. Nichols WW, O'Rourke MF: McDonald's blood flow in arteries: Theoretical, experimental and clinical principles. 5th edition. London: Hodder Arnold; 2005.

26. Mancia G, De Backer G, Dominiczak A, Cifkova R, Fagard R, Germano G, Grassi G, Heagerty AM, Kjeldsen SE, Laurent S, Narkiewicz K, Ruilope L, Rynkiewicz A, Schmieder RE, Boudier HA, Zanchetti A, Vahanian A, Camm J, De Caterina R, Dean V, Dickstein K, Filippatos G, Funck-Brentano C, Hellemans I, Kristensen SD, McGregor K, Sechtem U, Silber S, Tendera M, Widimsky P, et al: 2007 Guidelines for the Management of Arterial Hypertension: The Task Force for the Management of Arterial Hypertension of the European Society of Hypertension (ESH) and of the European Society of Cardiology (ESC). J Hypertens 2007, 25:1105-1187.

27. Altman R, Asch E, Bloch D, Bole G, Borenstein D, Brandt K, Christy W, Cooke TD, Greenwald R, Hochberg M, Howell D, Kaplan D, Koopman W, Longley S III, Mankin H, McShane DJ, Medsger T, Meenan RJR, Mikkelsen W, Moskowitz R, Murphy W, Rothschild B, Segal M, Sokoloff L, Wolfe F: Development of criteria for the classification and reporting of osteoarthritis. Classification of osteoarthritis of the knee. Diagnostic and Therapeutic Criteria Committee of the American Rheumatism Association. Arthritis Rheum 1986, 29:1039-1049.

28. Hochberg MC, Chang RW, Dwosh I, Lindsey S, Pincus T, Wolfe F: The American College of Rheumatology 1991 revised criteria for the classification of global functional status in rheumatoid arthritis. Arthritis Rheum 1992, 35:498-502.

29. Altman RD, Hochberg M, Murphy WA Jr, Wolfe F, Lequesne M: Atlas of individual radiographic features in osteoarthritis. Osteoarthritis Cartilage 1995, 3(Suppl A):3-70.

30. Wigg AE, Prest C, Slobodian P, Need AG, Cleland LG: A system for improving vitamin D nutrition in residential care. Med J Aust 2006, 185:195-198.

31. Bellamy N, Buchanan WW, Goldsmith CH, Campbell J, Stitt LW: Validation study of WOMAC: a health status instrument for measuring clinically important patient relevant outcomes to antirheumatic drug therapy in patients with osteoarthritis of the hip or knee. J Rheumatol 1988, 15:1833-1840

32. Peterfy CG, Guermazi A, Zaim S, Tirman PF, Miaux Y, White D, Kothari M, Lu Y, Fye K, Zhao S, Genant HK: Whole-Organ Magnetic Resonance Imaging Score (WORMS) of the knee in osteoarthritis. Osteoarthritis Cartilage 2004 12:177-190

33. Ding C, Martel-Pelletier J, Pelletier JP, Abram F, Raynauld JP, Cicuttini F, Jones G: Meniscal tear as an osteoarthritis risk factor in a largely non-osteoarthritic cohort: a cross-sectional study. J Rheumatol 2007, 34:776-784.

34. Hides J, Gilmore C, Stanton W, Bohlscheid E: Multifidus size and symmetry among chronic LBP and healthy asymptomatic subjects. Man Ther 2008, 13:43-49.

35. Hides JA, Miokovic T, Belavy DL, Stanton WR, Richardson CA: Ultrasound imaging assessment of abdominal muscle function during drawing-in of the abdominal wall: an intrarater reliability study. J Orthop Sports Phys Ther 2007, 37:480-486.

36. Koppenhaver SL, Hebert JJ, Fritz JM, Parent EC, Teyhen DS, Magel JS: Reliability of rehabilitative ultrasound imaging of the transversus abdominis and lumbar multifidus muscles. Arch Phys Med Rehabil 2009, 90:87-94.
37. Laurent S, Cockcroft J, Van Bortel L, Boutouyrie P, Giannattasio C, Hayoz D, Pannier B, Vlachopoulos C, Wilkinson I, Struijker-Boudier H: Expert consensus document on arterial stiffness: methodological issues and clinical applications. Eur Heart J 2006, 27:2588-2505.

38. Aoyagi Y, Shephard RJ: A model to estimate the potential for a physical activity-induced reduction in healthcare costs for the elderly, based on pedometer/accelerometer data from the Nakanojo Study. Sports Med 2011, 41:695-708.

39. Craig $C L$, Marshall AL, Sjostrom M, Bauman AE, Booth ML, Ainsworth BE, Pratt M, Ekelund U, Yngve A, Sallis JF, Oja P: International physical activity questionnaire: 12-country reliability and validity. Med Sci Sports Exerc 2003, 35:1381-1395.

40. Wang Y, Simpson JA, Wluka AE, Teichtahl AJ, English DR, Giles GG, Graves S, Cicuttini FM: Relationship between body adiposity measures and risk of primary knee and hip replacement for osteoarthritis: a prospective cohort study. Arthritis Res Ther 2009, 11:R31.

41. Cohen J: Statistical power analysis for the behavioral sciences. 2nd edition. New Jersey: Lawrence Erlbaum Associates, Inc; 1988.

42. Wluka AE, Stuckey S, Snaddon J, Cicuttini FM: The determinants of change in tibial cartilage volume in osteoarthritic knees. Arthritis Rheum 2002, 46:2065-2072.

43. Bischoff-Ferrari HA, Willett WC, Wong JB, Giovannucci E, Dietrich T, Dawson-Hughes B: Fracture prevention with vitamin D supplementation: a meta-analysis of randomized controlled trials. JAMA 2005, 293:2257-2264.

44. Wang $Y$, Wluka AE, Cicuttini FM: The determinants of change in tibial plateau bone area in osteoarthritic knees: a cohort study. Arthritis Res Ther 2005, 7:R687-R693.

45. Wluka AE, Ding C, Jones G, Cicuttini FM: The clinical correlates of articular cartilage defects in symptomatic knee osteoarthritis: a prospective study. Rheumatology (Oxford) 2005, 44:1311-1316.

46. Wang Y, Ding C, Wluka AE, Davis S, Ebeling PR, Jones G, Cicuttini FM: Factors affecting progression of knee cartilage defects in normal subjects over 2 years. Rheumatology (Oxford) 2006, 45:79-84.

47. Losina ECJ, Hunter DJ, Eckstein F, Nevitt MC, Cotofana S, Kwoh CK, Katz JN: The minimal clinically important difference (MCID) in cartilage volume and thickness change in persons with knee osteoarthritis[abstract]. Osteoarthritis Cartilage 2011, 19(Suppl 1):177-178.

48. Henrotin Y, Lambert C, Couchourel D, Ripoll C, Chiotelli E: Nutraceuticals: do they represent a new era in the management of osteoarthritis? - a narrative review from the lessons taken with five products. Osteoarthritis Cartilage 2011, 19:1-21.

49. Guermazi A, Hunter DJ, Roemer FW: Plain radiography and magnetic resonance imaging diagnostics in osteoarthritis: validated staging and scoring. J Bone Joint Surg Am 2009, 91(Suppl 1):54-62.

50. Cicuttini FM, Jones G, Forbes A, Wluka AE: Rate of cartilage loss at two years predicts subsequent total knee arthroplasty: a prospective study. Ann Rheum Dis 2004, 63:1124-1127.

51. Raynauld JP, Martel-Pelletier J, Haraoui B, Choquette D, Dorais M, Wildi LM, Abram F, Pelletier JP: Risk factors predictive of joint replacement in a 2-year multicentre clinical trial in knee osteoarthritis using MRI: results from over 6 years of observation. Ann Rheum Dis 2011, 70:1382-1388.

52. Holick MF, Binkley NC, Bischoff-Ferrari HA, Gordon CM, Hanley DA, Heaney RP, Murad MH, Weaver CM: Evaluation, treatment, and prevention of vitamin D deficiency: an Endocrine Society clinical practice guideline. J Clin Endocrinol Metab 2011, 96:1911-1930.

53. Chel V, Wijnhoven HA, Smit JH, Ooms M, Lips P: Efficacy of different doses and time intervals of oral vitamin D supplementation with or without calcium in elderly nursing home residents. Osteoporos Int 2008, 19:663-671.

54. Hyde J, Stanton WR, Hides JA: Abdominal muscle response to a simulated weight-bearing task by elite Australian Rules football players. Hum Mov Sci 2012, 31:129-138

55. Pilz S, Tomaschitz A, Ritz E, Pieber TR: Vitamin D status and arterial hypertension: a systematic review. Nat Rev Cardiol 2009, 6:621-630.

\section{doi:10.1186/1745-6215-13-131}

Cite this article as: Cao et al:: Vitamin D supplementation in the management of knee osteoarthritis: study protocol for a randomized controlled trial. Trials 2012 13:131. 